\title{
Quality of Control of Clinical-Biochemical Laboratories - Serbian Case
}

\author{
Vinko Peric ${ }^{1}$, Dusan Jaric ${ }^{1}$, Sonja Ketin ${ }^{2 *}$, Admira Konicanin ${ }^{3}$, Rade Biocanin ${ }^{3}$ \\ ${ }^{1}$ International University of Brcko, District Brcko, Bosnia and Herzegovina; ${ }^{2}$ University of Novi Sad, Novi Sad, Serbia; \\ ${ }^{3}$ University of Novi Pazar, Novi Pazar, Serbia
}

\begin{abstract}
Citation: Peric V, Jaric D, Ketin S, Konicanin A Biocanin R. Quality of Control of ClinicalBiochemical Laboratories - Serbian Case. OA Maced J Med Sci. 2014 Jun 15; 2(2):219-223. http://dx.doi.org/10.3889/oamjms.2014.034

Key words: medical laboratory; quality control; good laboratory practice; certification; accreditation.

"Correspondence: Sonja Ketin, PhD. Chemical Engineering, Technology at University of Belgrade, Environmental Engineering, Technical Science at University of Novi Sad, Novi Sad, Serbia. E-Mail: stefanov.sonja@gmail.com

Received: 07-Mar-2014; Revised: 24-Mar2014; Accepted: 09-May-2014; Online first: 06-Jun-2014

Copyright: () 2014 Peric et al. This is an open access article distributed under the terms of the Creative Commons Attribution License, the Creative Commons Atribution License, which permits un istribution, and reproduction in any medium, provide

Competing Interests: The authors have declared that no competing interests exist.
\end{abstract}

\begin{abstract}
In the last 20 years in medical laboratories, numerous activities regarding quality and accreditation system were taken. Approach to this problem in European countries is different, so the task of the Accreditation Work Group of the Confederation of European societies for clinical chemistry (EC 4) to help the efforts to harmonize this issue. External quality control in clinical-chemical laboratories imposed the need for the implementation of quality management system. "Good laboratory practice and its principles were adopted by nominated bodies, both international and national. In the beginning, the standard ISO 9001 was applied for certification and for accreditation EN 45001 and ISO Guide 25, which are prepared for testing and calibration laboratories. Standard ISO 17025 is the successor of the previous documents and for now it is a reference for mentioned laboratories. Accreditation Work Group of the Confederation of European societies for clinical chemistry (EC 4) made an amendment of the requirements for medical laboratories, which this standard describes. Standard draft ISO 15189 was adopted on February 2003 as a final version with requirements for medical laboratories.
\end{abstract}

\section{Introduction}

Clinical chemistry, by the application of chemical, molecular and cellular knowledge and techniques provides understanding and evaluation of health and illness of people. Measurement results enable discovery of the cause of illness and maintenance of health through specific and general information on relation laboratory-clinic. This discipline depends on good knowledge of fundamental and applied knowledge [1].

Laboratory must have its well-controlled technological and organizational principle both within laboratory, as well as towards health institution, i.e. patient and doctor who treats him. For that reason, technological and organizational principles of laboratory work must provide undisturbed and efficient flow of human biological material and information.

In the last 20 years in medical laboratories, numerous activities regarding quality and accreditation system were taken. Approach to this problem in European countries is different, so the task of the Accreditation Work Group of the Confederation of European societies for clinical chemistry (EC 4) to help the efforts to harmonize this issue. From the very beginning, European Diagnostic Manufacturers Associations, (EDMA) was invited to take a part in this process, having in mind that harmonization and accreditation of quality system are of common interest. Revolution in quality is reached in medical laboratories and it has a broader meaning even that quality management system itself, it is enabled by establishing the cooperation between manufacturers, laboratories, doctors and patients. Actually, quality of 
laboratory tests largely depends on products that come from industry on one hand and quality management in laboratories itself.

\section{Organization and control of the quality of clinical-chemical laboratories}

From organizational point of view, content of laboratory's work can be divided in three groups:

\section{- flow of biological material, \\ - data flow and \\ - analytical work}

Work in clinical-biochemical laboratories is organized in such a way that it includes several phases: reception of patients and samples, marking samples taken and examination status, triage of biological material, laboratory tests, and maintenance of work environment conditions and means of work, timely issuance of test results [2].

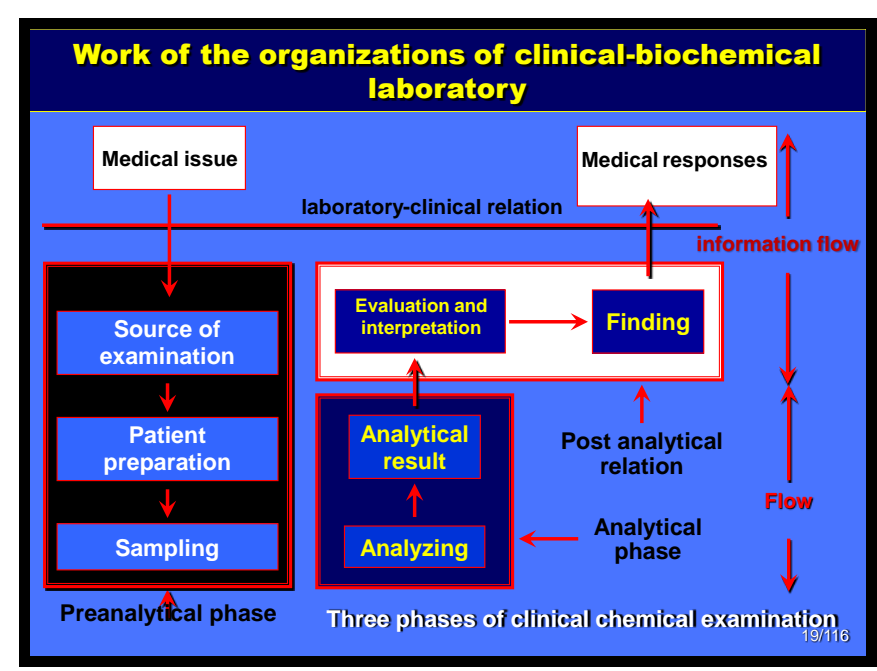

Figure 1: Work of the organizations of clinical-biochemical laboratory.

In the field of clinical-biochemical laboratory diagnosis today, great attention is paid to the concept of total quality in the laboratory process. At the international and national level it is approached to standardization of all phases of laboratory operations, total quality system (work processes management according to the management standards). External and internal quality control of the work and good laboratory practice it is ensureed that medical laboratories issue accurate and valid findings.

The need for implementing quality in laboratories has imposed the production of documents, which is adopted by the nominated bodies of experts in the field. Thus defined document is called regulation. Management of analytical quality of the performance of laboratory tests depends on welldefined quality standards. Laboratories voluntarily began with the introduction of the principle of "good laboratory practices" to improve their work. At the beginning of the seventies some governments formally introduced Good Laboratory Practice (GLP) as a means of control of laboratory activities.

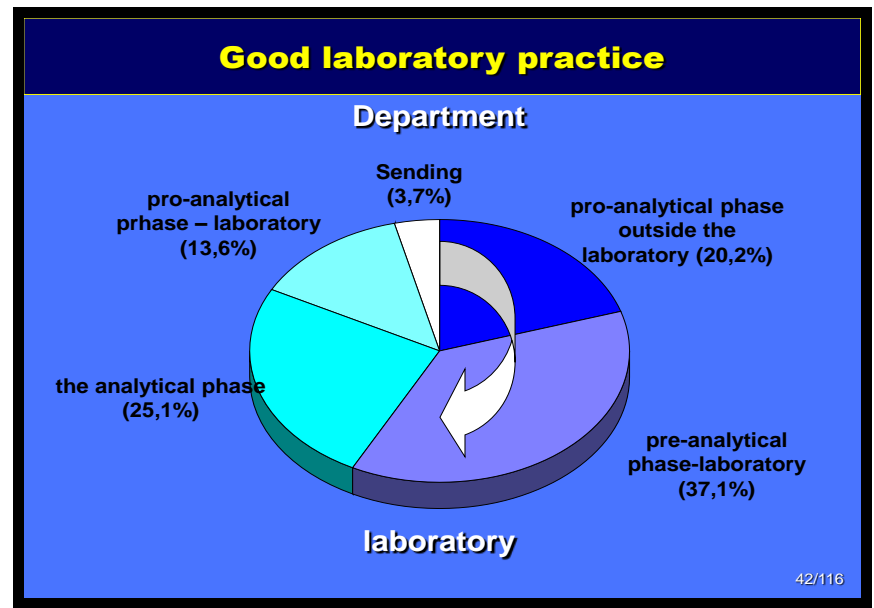

Figure 2: Good laboratory practice.

Introduction of Total Quality System (TQS) for medical and clinical laboratories and accrediting them is lately of particular importance and interest. Program of internal and external quality control includes only some of the degrees of laboratory work. However, complete control of all laboratory processes can be achieved only by applying total quality management system. As medical laboratories perform a specific type of laboratory activities, it was necessary to build an international system of quality criteria for use in this area.

International standards for the certification of ISO 9001, EN 45001 and ISO Guide 25 (ISO 17025) for accreditation, which are either very general or developed for laboratories that test or calibration, do not refer to specific aspects of working in medical laboratories. Successor of the document ISO Guide 25 and EN 45001 for testing laboratories, is the ISO document 17025 "General Requirements for the Competence of Testing and Calibrating Laboratories * [3].

As the activity of medical and clinical laboratories differs from the test ones, it was necessary for the documents to include additional criteria. In order to achieve this goal in a number of European countries for the purposes of laboratory medicine there were introduced quality systems and accreditation schemes.

The standards of these programs are similar, although there are certain differences. Therefore, in many countries, concerns arose over which system should be selected and implemented. In the European Union in matters of harmonization of quality systems and accreditation works EC4 Working Group, which was formed on the initiative of several members of the European Communities Confederation of Clinical Chemistry (EC4). One of the goals of EC4 is to work 
on the preparation and publication of the main criteria of quality systems in medical laboratories [4] Document "Essential Criteria for Quality Systems of Medical Laboratories * [5] clarifies the specific needs of the biochemical laboratory.

Publication EC4 Essential Criteria stimulated ISO to adopt the International Standard 15189, entitled "Medical laboratories - Particular requirements for quality and competence" * [6], which is more suitable for medical laboratories. ISO 15189 is a very important document for medical laboratories, which will help this type of laboratory to develop a quality system in a harmonized way. Significantly, ISO 15189 fully covers the needs defined by ISO 9001 : 2000. Difficulties about its interpretation are dismissed by the application of EC4 Essential Criteria.

All of these documents enabled for the entire work process, from collecting and preparing specimens (preanalytical phase) to issueof laboratory results (postanalytical phase), to be fully defined, standardized, and controlled in order to obtain valid laboratory results. Results of laboratory testing, i.e. response to a specific question of the doctors is a lab result, which is not just a simple result of chemical analize. Behind each results stands a multilayer test procedures of the laboratory work, so that found result could became the laboratory finding. Verification involves three successive degrees marked as:

- $\quad$ technical« (analytical result),

- «biological« (finding) and

- nosological« (finding interpretation) phase

In the "Technical" level biochemist evaluates and selects the appropriate method by applying quality control, analytical evaluation and assessment of the various impacts. The "biological level" estimates number of biological effects (eg., age, gender, race, etc.) as intra-and inter-individual variations;

In teh domain of so-called "Transversal estimates" the obtained result is compared to the reference population, and then the result is declared as "pathological" or "non-pathological." Laboratory findings are usually submitted in a written report which must contain all the required information (eg., value, unit, reference values, etc.). For more complex tests, such as coagulation tests, drug levels in the blood, molecular-biological testing, etc., in addition to the written report, the laboratory should establish cooperation with a doctor at the level of verbal consultations. Responses to requests for physician for the best laboratory findings are contained in the application of Good Laboratory Practice (GLP).

The service of a good clinical laboratory is cahracterized by technical requirements (availability according to the medical needs, analysis production speed, the application of a small amount of the samples according to medical need, the accuracy of the results according to medical needs, report on the indication of the uncertainty of method) and medical (probability control, information on the validity of the applied methods (diagnostic, prognostic), appropriate findings according to clinical state, the interpretation of the biological level, accurate reference values, comparability with previous results, aid in the interpretation of the results at the nosological level, dialogue with clinicians, consultations).

To establish a solid relationship of the laboratory with clinicians, the laboratory work must be improved on daily basis including: continuous training of personnel, implementation of continuous control (analytical processes, tools, methods, introducing a system of total quality management), data collection (on biological influences, other interferences, reference intervals, validation procedures, etc.)..

Since most of the errors occur in the pre-and post-analytical phase of laboratory work, quality control and improvement of pre-analytical phase is crucial for obtaining good laboratory findings. For that purpose, it is necessary to define the procedures to be implemented in the diagnostic process between patient and analytical grade. It is necessary to define a set of requirements relating to biological samples such as: adequate sample size, the use of anticoagulants and stabilizers, sampling time, transport, storage in accordance to teh stability of the sample, the corresponding pre-analytical treatment.

In the instructions that refer to the preanalytical phase, it is required to standardize the following operations: 1) identification of the sample, 2) storage, 3) interference and influence of many factors on the measured parameter.

Biological sample must be properly sampled. The correct pattern is one of the conditions for the lab results to be accurate. Each health facility has rules and regulations regarding the collection and transportation of samples to the laboratory. In order to obtain the correct sample, sometimes the preparation of the patient is required as is the case of the determination of lipid status, which the patient needs to be informed about firstly. The laboratory must provide the doctors and nurses with all necessary instructions used by patients, for example, regarding the diet, the method of collecting certain materials etc. The sample should be marked with printed labels. It is important for the sample to be reliably marked and that the label can not be easily removed, allowing the samples to remain unmarked.

Sampling is done according to the referral that the patient receives from physician. Referral enables communication between doctors, patients and laboratories. Referrals used in practice may be different in form and content but they all have the same function for identifying in the laboratory what must be determined in referred biological sample. Laboratory conceives referral according to teh needs of healthcare facility. 
All data from the referral on the patient, biological samples, and then the obtained results of examination are entered in the laboratory protocol. Laboratory protocol is the main and most important official document in the laboratory. It is also the central point from which the control of laboratory work by specialized departments of the parent institution begins. This especially applies to the services of legal and financial affairs.

Laboratoria: Occupational Medicine „14.October“, Krusevac, Serbia Parameter: Creatine

Unit: U/L

Your methods: $\beta$ alkaline picrate. (Jaffe), without deproteinization Your result: 478.00

$(+)-$ Your method (0) - Others method $\left(^{*}\right)-$ Your result

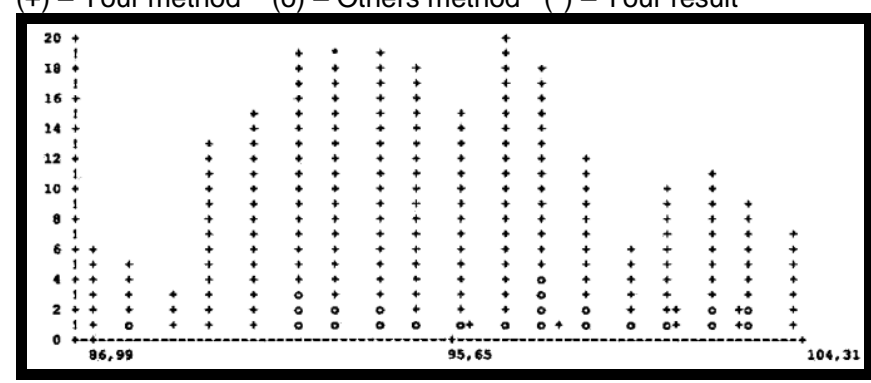

Figure 3: Result for Creatine.

In addition to the internal control of the laboratory work (control of technological processes, carried out by specialists in medical biochemistry and control of legal and financial services of health facilities), external quality control of biochemical laboratories is also carried out. The introduction of these controls has enabled many laboratories to improve the quality of their work by strengthened internal control, as well as pressure on the management structures to buy the best possible equipment and reagents. So today most laboratories in Serbia have at least one automatic analyzer. Automation has increased the productivity of laboratories' work, reductions in staff and the cost of reagents, and consequently the cost of health care. Most modern automatic analyzers includesone or more microprocessors that perform different functions. These functions include the control of the analyzer, the transmission of information (requirements, manipulation, verification and storage), communication between the operator and the analyzer, publishing of the results (directly or through an independent computer). Progress in the development of new diagnostic tests and automated analyzers in the last 20 years has led to a sharp increase in the number of analyzes done in clinical laboratories. With the increase of the determinations number, number of information whose processing imposes the development of Laboratory Information Systems (LIS) aslo increases. LIS includes sophisticated computers and computer software, which needs to satisfy the specific needs of clinical laboratories and other health care systems.
Table 1: National program for external quality SNEQASmedical - biochemical for result for creatine.

\begin{tabular}{lcc}
\hline & Yours methods & All methods \\
\hline Number of good results & 28 & 167 \\
Agreement of velue & 467.12 & 466.5 \\
Lower and upper bound & 430.22 & \\
results & 504.02 & 439.67 \\
Lower and upper bound & & 493.35 \\
Standard deviation & 14.38 & 13.42 \\
Coefficient of variation & 3.08 & 2.88 \\
Index standard deviation & 0.76 & \\
Calculation of the index & & \\
standard deviation & & \\
Total number of results & 41 & \\
\hline
\end{tabular}

And finally, here's an example of external quality control (SNEQAS) which is implemented by the Society of Medical Biochemists of Serbia - a national program of external quality control. Program manager for quality control and accreditation is prof. $\mathrm{PhD}$ Svetlana Ignjatovic. The organizer of the quality control sends lyophilised serum to all laboratories participating in the program. That serum should be dissolved on the day the organizer indicated in the accompanying documentation when sending it. Upon dissolution of serum, all parameters in it for which the laboratory has appropriate equipment and determination methodology are determined. The results obtained are entered into the appropriate questionnaire, which the organizer also forwards to the participants in the field of External Quality Control. Participant writes the obtained value for each parameter, the method that is used to determine it, as well as manufacturers of reagents used in this method. Thus entered results are sent to the organizer, who upon receipt statisticaly analyzes them and then sends them back and to each laboratory. The enclosure contains a scan of two such responses. In Figure 3 and 4 show graphically the results Society of Medical Biochemists Serbian - National quality control program of the parameters creatine and alanine aminotransferase. In Tables 1 and 2 values are shown in National program for external quality SNEQAS-medical - biochemical for result for creatine and alanine aminotransferase.

Laboratoria: Occupational Medicine „14.October“, Krusevac, Serbia Parameter: Alanine aminotransferase

Unit: U/L

Your methods: A optimized, Tris buffer, without the PP, (IFCC/SFBC)

Your result: 93.00

$(+)$ - Your method (o) - Others method $\left(^{*}\right)-$ Your result

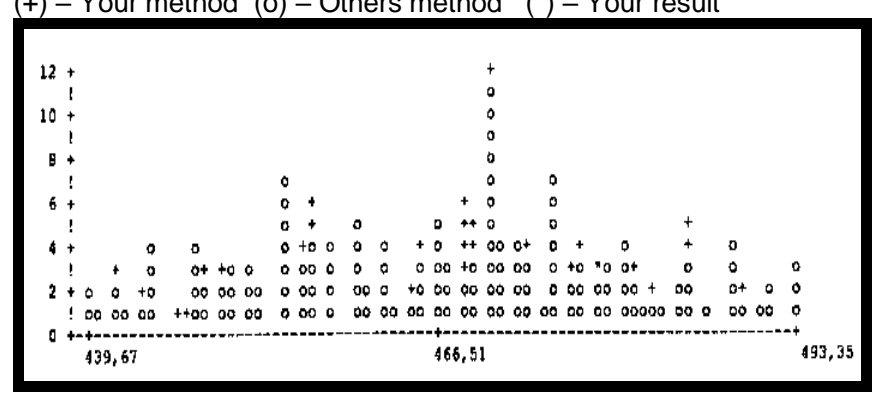

Figure 4: Result for Alanine aminotransferase. 
For a clinician, good laboratory is teh one that promptly prepares and submits laboratory analysis. In order to form such a clinical- biochemical laboratory, it is necessary to provide: adequate space according to the surface and purpose, staff with adequate qualifications, equipment, supplies and planned organization and work technology according to the needs of healthcare facility. In a suchlaboratory, errors must be reduced to a minimum. It is well known that most laboratory errors occur in the pre-and postanalytical phases of the laboratory work, and that only a small number of errors can occur during the current analytical phase. On the other hand, total quality management in medical laboratories enables the improvement of all levels of laboratory work, in order to eliminate the defects in the total testing process, including pre-and post-analytical phase of the work. Cooperation with clinicians and other personnel outside the laboratory, including producers, is crucial for the improvement of overall quality. User needs include several elements that encourage the producers to reach analytical quality.

Table 2: National program for external quality SNEQASmedical - biochemical for result for alanine aminotransferase.

\begin{tabular}{lcc}
\hline & Yours methods & All methods \\
\hline Number of good results & 208 & 231 \\
Agreement of velue & 95.52 & 95.65 \\
Lower and upper bound & 76.42 & \\
results & 114.62 & 86.99 \\
Lower and upper bound & & 104.31 \\
& & 4.33 \\
Standard deviation & 4.34 & 4.53 \\
Coefficient of variation & 4.54 & \\
Index standard & 0.58 & \\
deviation & & \\
Calculation of the index & 1.67 & 283 \\
standard deviation & & \\
Total number of results & 249 & \\
\hline
\end{tabular}

Manufacturers of in vitro diagnostic (IVD) medical devices and laboratory management have become integral partners in building and improving the quality of laboratory service. It has become abundantly clear that the quality comes from the structure of a reagent or analytical system. In vitro diagnostic medical devices should provide patients, users and any other party a high level of health care. Thus, manufacturers and users must have a permanent cooperation in order to improve the product. As you know, from day to day, the pressure on the standard of health services increases, particularly in terms of cost control, especially in medical laboratories. Medical laboratory services are essential to patient care. The most important aspects of quality in the health system are: patient care, prevention, treatment as soon as possible, risk reduction, use of teh advantages of new technologies, reducing the cost of treatment and time.

\section{Reference}

1. Maksimović R. Kliničko laboratorijska dijagnostika. Panevropski univerzitet Apeorin. Banja Luka, 2010

2. Majkic-Singh N, Đurđević J, Kavarić J. Razvoj medicinske biohemije u Jugoslaviji. Društvo medicinskih biohemičara Jugoslavije, 1998.
3. International Organisation for Standardization (ISO), General Requirements for the Competence of Testing and Calibrating Laboratories. (ISO 17025). ISO: Geneva.

4. International Organisation for Standardization (ISO), Medical laboratories Particular requirements for quality and competence (ISO 15189), ISO: Geneva.

5. Jansen RTP, Kenny D, Blaton V, Burnett D, Huisman W, Plebani $M$, Wueralto JM, Zerah S, van Lieshout J. Usefulness of EC4 Essential Criteria for Quality Systems of Medical Laboratories as Guideline to the ISO 15189 and ISO 17025 Documents. Clin Chem Lab Med 2000; 38: 105764.

6. Jansen RTP, Blaton V, Burnett D, Huisman W, Queralto JM, Zerah S, Allman B. European Communites Confede 7 ration of Clinical Chemistry: Essential Criteria for Quality Systems of Medical Laboratories. Eur J Clin Chem Bio-chem. 1997; 35: 123-132. 